\title{
Global bounds on heavy neutrino mixing
}

\section{Jacobo López-Pavón*}

CERN, Theoretical Physics Department, Geneva, Switzerland

E-mail: jacobo.lopez.pavon@cern.ch

\begin{abstract}
General bounds on the mixing between heavy and active neutrinos from a complete set of 28 observables are derived. Two scenarios are analysed and compared: (i) a general scenario in which an arbitrary number of heavy neutrinos is integrated out without any further assumption; (ii) only three heavy neutrinos are included and the light neutrino masses and mixings as measured in neutrino oscillations experiments are generated. A $1-2 \sigma$ preference for non-zero heavy mixing is founded and implies some interesting correlations in the more constrained scenario (ii). At the $2 \sigma$ level, the bounds on all the squared mixings are extremely similar in both scenarios. If only Lepton Flavour Conserving processes are included, they range from $\mathscr{O}\left(10^{-2}\right)$ to $\mathscr{O}\left(10^{-4}\right)$. Adding Lepton Flavour Violating observables as $\mu \rightarrow e \gamma$ to the analysis improves the bound in the $\mu-e$ sector by one order of magnitude.
\end{abstract}

Neutrino Oscillation Workshop

4 - 11 September, 2016

Otranto (Lecce, Italy)

${ }^{*}$ Speaker. 


\section{Introduction}

The recent discovery of the Higgs boson has partially completed the mass generation puzzle. However, the origin of the light neutrino masses is still a completely open question. The simplest model which can account for the light neutrino masses consists in the addition of fermion singlets to the Standard Model (SM) field content [1]. The new states participate in the neutrino mass generation and mix with the SM neutrinos. In this work we will consider 28 relevant flavour and electroweak (EW) observables which can be affected by this type of New Physics (NP) in order to constrain the heavy mixing. We will assume that the heavy neutrinos are heavier than the EW scale and therefore integrated out. The NP effects are then driven by the $d=5$ and $d=$ 6 non-renormalizable effective operators which should be added to the SM Lagrangian. After the EW symmetry breaking, the $d=5$ Weinberg operator [2] generates the light neutrino masses while the $d=6$ operator [3] induces deviations from the unitarity of the leptonic mixing matrix $N$ $[4,5,6,7,8]$ which can be parametrized with all generality as [9]

$$
N=(1-\eta) U_{P M N S},
$$

where $U_{P M N S}$ is a unitary matrix which reassembles the standard PMNS matrix up to the small non-unitarity corrections and

$$
\eta=\frac{\Theta \Theta^{\dagger}}{2}
$$

is the coefficient of the $d=6$ operator and it is directly related to the heavy-active mixing $\Theta_{\alpha j}$.

Two scenarios will be studied:

(i) G-SS. A general scenario in which an arbitrary number of heavy neutrinos is included without any further assumption.

(ii) $\mathbf{3 N}$-SS. The 3 right-handed neutrino case defined by the following requirements:

- The SM is only extended adding 3 right-handed neutrinos.

- Potentially observable $\eta$ allowed despite the smallness of neutrino masses.

- The light neutrino masses are radiatively stable [10, 11].

In order to satisfy these conditions the Dirac and Majorana mass matrices should show some particular textures which can be understood in an approximate Lepton number $(L)$ conserving context ${ }^{1}$. Indeed, since the smallness of the neutrino masses can come not only from the new physics scale suppression but also from an underlying approximate symmetry [12] such as $L$, the mixings can be much larger than the ones expected in the naive seesaw scaling, $\mathscr{O}\left(m_{v} / M\right)$, and saturate the present experimental bounds. In this $3 \mathrm{~N}-\mathrm{SS}$ scenario the heavy mixing pattern simplifies at leading order to

$$
\eta=\frac{\Theta \Theta^{\dagger}}{2}=\frac{1}{2}\left(\begin{array}{ccc}
\left|\theta_{e}\right|^{2} & \theta_{e} \theta_{\mu}^{*} & \theta_{e} \theta_{\tau}^{*} \\
\theta_{\mu} \theta_{e}^{*} & \left|\theta_{\mu}\right|^{2} & \theta_{\mu} \theta_{\tau}^{*} \\
\theta_{\tau} \theta_{e}^{*} & \theta_{\tau} \theta_{\mu}^{*} & \left|\theta_{\tau}\right|^{2}
\end{array}\right) \quad \text { with } \quad \theta_{\alpha} \equiv \frac{Y_{N \alpha} \nu}{\sqrt{2} \Lambda},
$$

where $v$ is the Higgs vev and $\Lambda$ is the $3 \mathrm{~N}-\mathrm{SS}$ scale (the mass of the Dirac heavy neutrino pair when the symmetry is exact).

\footnotetext{
${ }^{1}$ See [8] for details.
} 


\section{Set of Observables}

The global constraints to the heavy-active mixing have been obtained through the fit to the following 28 observables written as a function of $\alpha, M_{Z}$ and the Fermi constant as measured from the muon decay $G_{\mu}$ :

-The $W$ boson mass $M_{W}$

- The effective weak mixing angle $\theta_{\mathrm{W}}: s_{\mathrm{W} \text { eff }}^{2 \text { lep }}$ and $s_{\mathrm{W} \text { eff }}^{2 \text { had }}$

- Four ratios of $Z$ fermionic decays: $R_{l}, R_{c}, R_{b}$ and $\sigma_{\text {had }}^{0}$

- The $Z$ invisible width $\Gamma_{\text {inv }}$

- Weak decays ratios constraining EW universality: $R_{\mu e}^{\pi}, R_{\tau \mu}^{\pi}, R_{\mu e}^{W}, R_{\tau \mu}^{W}, R_{\mu e}^{K}, R_{\tau \mu}^{K}, R_{\mu e}^{l}$ and $R_{\tau \mu}^{l}$

- 9 weak decays constraining the CKM unitarity

- 3 radiative Lepton Flavour Violating (LFV) decays: $\mu \rightarrow e \gamma, \tau \rightarrow \mu \gamma$ and $\tau \rightarrow e \gamma$

The details of the analysis can be found in [8], but let us briefly discuss the sensitivity of the LFV observables to $\eta_{\alpha \beta}$. In Fig. 1 we show the current experimental bounds and future sensitivities to $\eta_{e \mu}$ and $\eta_{e \tau}$. The present bound is currently dominated by the radiative decays $\mu \rightarrow e \gamma$ and $\tau \rightarrow e \gamma$ (this is also the case in the $\mu-\tau$ sector with $\tau \rightarrow \mu \gamma$ ). Notice that $\mu-e$ conversion and $\mu \rightarrow$ eee already give quite competitive bounds at present while in the future they will have the strongest sensitivity in the $\mu-e$ sector, being able to probe $\eta_{e \mu}$ values even three orders of magnitude smaller than the current constraint.
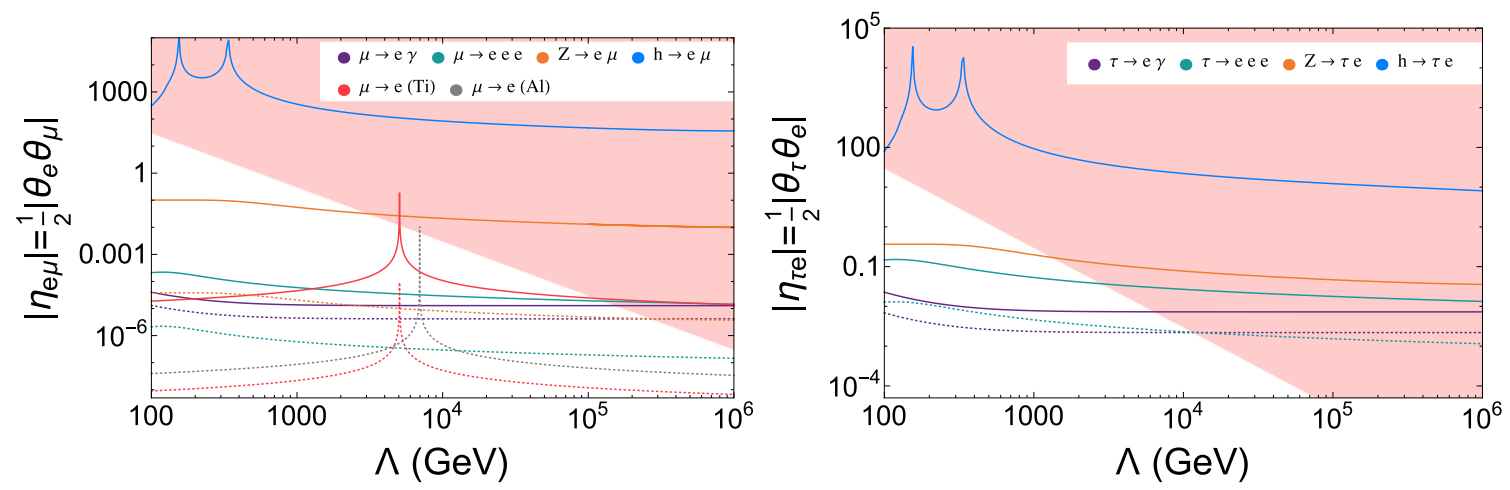

Figure 1: $90 \% \mathrm{CL}$ constraints on $\eta_{e \mu}$ (left panel) and $\eta_{e \tau}$ (right panel) from LFV observables in the $3 \mathrm{~N}-$ SS. Solid lines represent current experimental bounds while dotted lines represent future sensitivities. The red-shadowed region is the region with $\left|Y_{N}\right|^{2}>6 \pi$.

Since $\eta$ is a positive-definite matrix (see eq. (1.2)), its non diagonal elements should satisfy the Schwarz inequality

$$
\left|\eta_{\alpha \beta}\right| \leq \sqrt{\left|\eta_{\alpha \alpha}\right|\left|\eta_{\beta \beta}\right|}
$$

This is a key point since, as we will see in the next section, only the bound on $\eta_{e \mu}$ is dominated by the current LFV constraint.

\section{Global bounds}

The results of our global fit to the Lepton Flavour Conserving (LFC) observables are shown in Fig. 2 for the G-SS (top panels) and the 3N-SS with inverted hierarchy (bottom panels). The 


\begin{tabular}{|c|c|c|c|c|c|c|c|}
\cline { 3 - 8 } \multicolumn{2}{c|}{} & $\sqrt{2 \eta_{e e}}$ & $\sqrt{2 \eta_{\mu \mu}}$ & $\sqrt{2 \eta_{\tau \tau}}$ & $\sqrt{2 \eta_{e \mu}}$ & $\sqrt{2 \eta_{e \tau}}$ & $\sqrt{2 \eta_{\mu \tau}}$ \\
\hline \multirow{2}{*}{ LFC } & $1 \sigma$ & $\mathbf{0 . 0 3 1}_{-\mathbf{0 . 0 2 0}}^{+\mathbf{0 . 0 1 0}}$ & $<\mathbf{0 . 0 1 1}$ & $\mathbf{0 . 0 4 4}_{-\mathbf{0 . 0 2 7}}^{+0.019}$ & $<0.018$ & $<\mathbf{0 . 0 4 5}$ & $<\mathbf{0 . 0 2 4}$ \\
\cline { 2 - 8 } & $2 \sigma$ & $<\mathbf{0 . 0 5 0}$ & $<\mathbf{0 . 0 2 1}$ & $<\mathbf{0 . 0 7 5}$ & $<0.026$ & $<\mathbf{0 . 0 5 2}$ & $<\mathbf{0 . 0 3 5}$ \\
\hline \multirow{2}{*}{$\mathrm{LFV}$} & $1 \sigma$ & - & - & - & $<\mathbf{4 . 1} \cdot \mathbf{1 0}^{-3}$ & $<0.107$ & $<0.115$ \\
\cline { 2 - 8 } & $2 \sigma$ & - & - & - & $<\mathbf{4 . 9}^{-10} \mathbf{1 0}^{-3}$ & $<0.127$ & $<0.137$ \\
\hline
\end{tabular}

Table 1: 1 and $2 \sigma$ bounds on non-unitarity (heavy-active neutrino mixing). For the off-diagonal entries the indirect bounds from the LFC observables via the Schwarz inequality eq. (2.1) are compared with the direct LFV bounds and the dominant bound is highlighted in bold face.

bounds in the $3 \mathrm{~N}$-SS with normal hierarchy are similar to the inverted hierarchy case and can be found in [8]. The generation of the the light neutrino mass and mixing in the 3N-SS implies nontrivial correlations which are not present in the the G-SS scenario as it can be observed comparing the top and bottom panels. However, the $2 \sigma$ individual bounds on $\eta_{\alpha \alpha}$ are pretty similar in both scenarios in spite of the stronger correlations present in the $3 \mathrm{~N}-\mathrm{SS}$. Notice that the data show a preference for non zero heavy-active mixing at $1 \sigma$.

Finally, we summarize our results for the G-SS scenario (in other words, the current nonunitarity bounds obtained in our analysis) in Table 1. It is remarkable that the indirect bounds on $\eta_{e \tau}$ and $\eta_{\mu \tau}$ from LFC observables are stronger than the direct LFV bounds from the corresponding radiative decays. Only the constraint on $\eta_{e \mu}$ is dominated by the LFV decay $\mu \rightarrow e \gamma$ and its LFC bound is already at the level of $\mathscr{O}\left(10^{-4}\right)$ in any case. Our bounds can be translated to the triangular $\alpha$ parameterization [13, 14] just using the mapping given in [8] as shown in [15].

\section{References}

[1] P. Minkowski, Phys. Lett. 67B (1977) 421; R. N. Mohapatra and G. Senjanovic, Phys. Rev. Lett. 44 (1980) 912; T. Yanagida, Conf. Proc. C 7902131 (1979) 95; M. Gell-Mann, P. Ramond and R. Slansky, Conf. Proc. C 790927(1979) 315.

[2] S. Weinberg, Phys. Rev. Lett. 43 (1979) 1566.

[3] A. Broncano, M. B. Gavela and E. E. Jenkins, Phys. Lett. B 552 (2003) 177; Erratum: Phys. Lett. B 636 (2006) 332.

[4] P. Langacker and D. London, Phys. Rev. D 38 (1988) 886.

[5] E. Nardi, E. Roulet and D. Tommasini, Phys. Lett. B 327 (1994) 319.

[6] S. Antusch, C. Biggio, E. Fernandez-Martinez, M. B. Gavela and J. Lopez-Pavon, JHEP 0610 (2006) 084.

[7] S. Antusch and O. Fischer, JHEP 1410 (2014) 094.

[8] E. Fernandez-Martinez, J. Hernandez-Garcia and J. Lopez-Pavon, JHEP 1608 (2016) 033.

[9] E. Fernandez-Martinez, M. B. Gavela, J. Lopez-Pavon and O. Yasuda, Phys. Lett. B 649 (2007) 427.

[10] J. Lopez-Pavon, S. Pascoli and C. F. Wong, Phys. Rev. D 87 (2013) no.9, 093007.

[11] E. Fernandez-Martinez, J. Hernandez-Garcia, J. Lopez-Pavon and M. Lucente, JHEP 1510 (2015) 130. 
[12] R. N. Mohapatra and J. W. F. Valle, Phys. Rev. D 34 (1986) 1642; J. Bernabeu, A. Santamaria, J. Vidal, A. Mendez and J. W. F. Valle, Phys. Lett. B 187 (1987) 303; G. C. Branco, W. Grimus and L. Lavoura, Nucl. Phys. B 312 (1989) 492; M. Malinsky, J. C. Romao and J. W. F. Valle, Phys. Rev. Lett. 95 (2005) 161801; J. Kersten and A. Y. Smirnov, Phys. Rev. D 76 (2007) 073005; M. B. Gavela, T. Hambye, D. Hernandez and P. Hernandez, JHEP 0909 (2009) 038.

[13] Z. Z. Xing, Phys. Lett. B 660 (2008) 515; Z. Z. Xing, Phys. Rev. D 85 (2012) 013008.

[14] F. J. Escrihuela, D. V. Forero, O. G. Miranda, M. Tortola and J. W. F. Valle, Phys. Rev. D 92 (2015) no.5, 053009; Erratum: Phys. Rev. D 93 (2016) no.11, 119905.

[15] M. Blennow, P. Coloma, E. Fernandez-Martinez, J. Hernandez-Garcia and J. Lopez-Pavon, arXiv:1609.08637 [hep-ph]. 

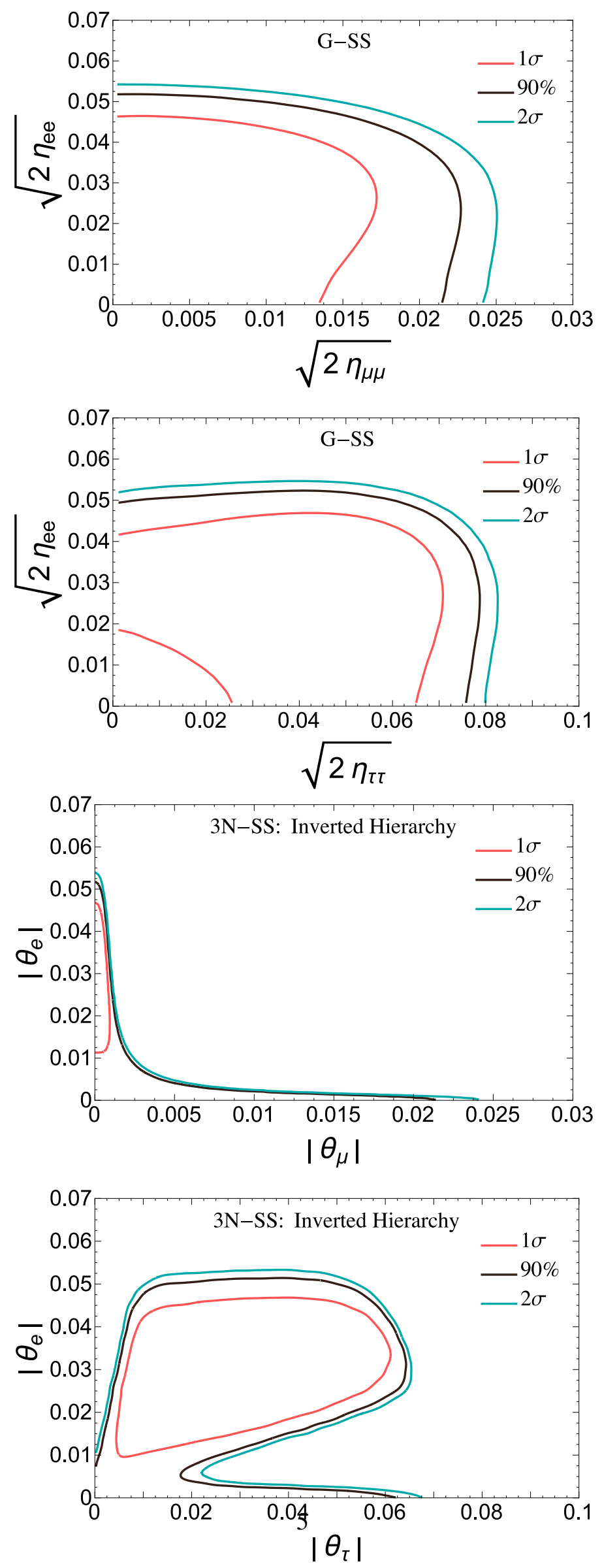

Figure 2: Frequentist confidence intervals at $1 \sigma, 90 \%$ and $2 \sigma$ on the parameter space of the G-SS and the $3 \mathrm{~N}-\mathrm{SS}$ for inverted hierarchy. 